\title{
MODELAGEM MATEMÁTICA COMO METODOLOGIA DE ANÁLISE DO CRESCIMENTO E ARQUITETURA DE SISTEMAS RADICULARES ${ }^{1}$
}

\author{
Dioger Alexandre Teruel2,6* ; Durval Dourado-Neto ${ }^{3,7} ;$ Jan W. Hopmans ${ }^{4}$; Klaus Reichardtt ${ }^{5,7}$ \\ ${ }^{2}$ Pós-Graduando do Depto. de Produção Vegetal - USP/ESALQ. \\ ${ }^{3}$ Depto. de Produção Vegetal - USP/ESALQ, C.P. 9 - CEP: 13418-900 - Piracicaba, SP. \\ ${ }_{5}^{4}$ Dept. of Land, Air and Water Resources - University of California, Davis, USA. \\ ${ }^{5}$ Depto. de Ciências Exatas - USP/ESALQ. \\ ${ }_{7}^{6}$ Bolsista FAPESP. \\ Bolsista CNPq. \\ *Autor correspondente<teruel@sum.desktop.com.br>
}

RESUMO: Devido à dificuldade de observação, quantificação e interpretação de sistemas radiculares, o estado da arte do conhecimento científico nesta área está aquém do desejável. Estas dificuldades tornam a análise e a interpretação da arquitetura de sistemas radiculares praticamente impossíveis de serem realizadas pela estatística tradicional, fazendo com que a modelagem matemática seja uma abordagem atrativa. Utilizou-se neste estudo plantas de soja, visto que a maioria dos trabalhos de pesquisa sobre a estrutura e funcionamento de sistemas radiculares de culturas comerciais tem sido realizada com um pequeno número de espécies, com ênfase nas monocotiledôneas, sendo muito poucos aqueles com dicotiledôneas herbáceas e espécies arbóreas. Estudou-se a influência da concentração de fósforo, nutriente freqüentemente deficiente nos solos brasileiros, sobre a arquitetura do sistema radicular da soja. A modelagem matemática apresentou-se como uma promissora metodologia de análise de dados de sistemas radiculares, possibilitando uma adequada visualização de diferentes estratégias adaptativas da planta, conforme refletidas pelas diferentes arquiteturas radiculares produzidas em cada condição.

Palavras-chave: Glycine max, modelo matemático, raiz, nutriente, fósforo

\section{MATHEMATICAL MODELING AS A TOOL FOR THE ANALYSIS OF GROWTH AND ARCHITECTURE OF ROOT SYSTEMS}

\begin{abstract}
Root systems are difficult to observe, quantify, and interpret, being those the reasons why the scientific knowledge about roots is so poor. These difficulties make root architecture virtually impossible to be properly analyzed and understood by classical statistics, being simulation modeling an attractive approach. The plant species studied in this paper was soybean, a herbaceous dicotyledon, since most of the structural and functional investigations on root systems have been carried out for a few monocotyledon species. Insufficient phosphorus supply in agricultural soils often affects crop yield, and therefore the effects of soil P concentration on soybean root architecture were studied. The proposed simulation model is a promising methodology for root data analysis, allowing a proper visualization of different plant adaptation strategies, as reflected by different root architectures.
\end{abstract}

Key words: Glycine max, modeling, mathematical model, root, soil nutrient, phosphorus

\section{INTRODUÇÃO}

É premente a necessidade de abordagens criativas no estudo de sistemas radiculares. Além das dificuldades de observação e quantificação de raízes, elas constituem um sistema bastante complexo, composto de milhares de segmentos individuais, de diferentes classes morfológicas, fisiológicas e de desenvolvimento.

Segundo Lynch (1995), não existe nenhuma estrutura analítica satisfatória ou ferramentas quantitativas adequadas para sumariar e caracterizar essa complexidade do sistema radicular, ainda mais visto que 0 crescimento e a arquitetura radicular variam amplamente devido à interação dinâmica com um grande número de fatores físicos, químicos e biológicos no solo, fatores estes também variáveis no espaço e no tempo. Estas dificuldades fazem com que a estatística clássica seja inadequada para a análise e a interpretação da arquitetura de sistemas radiculares, e tornam a modelagem matemática uma abordagem atrativa (Lynch, 1995).

A modelagem de sistemas radiculares como objetos geométricos dinâmicos tem apresentado um grande avanço nos últimos anos, como constatado pela evolução de tais modelos, desde os trabalhos pioneiros de Hackett \& Rose (1972) e de Lungley (1973), passando pelos de Rose (1983) e de Porter et al. (1986), até chegar aos modelos mais recentes, de Diggle (1988), Pages et al. (1989), Clausnitzer \& Hopmans (1993), Lynch et al. (1997) e Somma et al. (1998), entre outros. 
No entanto, até o momento muito pouco é conhecido dos processos fisiológicos que promovem uma determinada arquitetura radicular, e apesar dos progressos recentes, a modelagem das implicações funcionais da arquitetura radicular (p. ex. interações entre arquitetura radicular e eficiência de absorção de água e nutrientes) ainda está no estágio de desenvolvimento metodológico, e ainda não é de forma alguma uma ferramenta completamente validada e utilizável para previsão (Lynch, 1995).

Robinson (1991) enfatiza que o avanço científico no entendimento de como as raízes interceptam e absorvem os nutrientes seria muito pequeno se se tentasse reconstruir com exatidão detalhada, às custas de muito tempo, esforços e recursos financeiros despendidos, a intrincada estrutura tridimensional de um sistema radicular real. Novas idéias e abordagens criativas são mais importantes que avanços tecnológicos que permitam uma caracterização mais detalhada do sistema radicular, e sua exata reconstrução através de modelos de simulação. Sistemas radiculares devem ser simplificados conceitualmente, e a modelagem matemática pode ser utilizada como uma excelente ferramenta de síntese.

A modelagem e a simulação têm valor heurístico, ajudando o pesquisador a formular hipóteses sobre processos e interações relevantes, a quantificar o impacto de variáveis simples sobre o desempenho do sistema, e a sugerir novas necessidades de experimentação (Wullschleger et al., 1994)

Plantas de soja foram utilizadas neste estudo, visto que a maioria dos trabalhos de pesquisa sobre a estrutura e funcionamento de sistemas radiculares de culturas comerciais tem sido realizada com um pequeno número de espécies de monocotiledôneas, estando as dicotiledôneas herbáceas e as espécies arbóreas ainda muito pouco estudadas (McCully, 1995).

Experimentos preliminares realizados pelos autores, e dados na literatura (Drew, 1975; Robinson, 1994) mostraram um efeito pronunciado do conteúdo de alguns nutrientes no solo, especialmente o nitrogênio e o fósforo, sobre a arquitetura de sistemas radiculares. Os experimentos preliminares mostraram uma tendência para diminuição na densidade de ramificação, isto é, raízes laterais mais espaçadas e em ângulos próximos da vertical, em condições de baixos níveis de nitrogênio e fósforo no solo; por outro lado, em solos ricos nestes nutrientes verificou-se tendência de aumento na densidade de ramificação, isto é, raízes laterais em maior número numa determinada camada de solo e em ângulos próximos da horizontal.

Sendo rara a deficiência de nitrogênio na cultura da soja, devido à prática de inoculação de sementes com a bactéria fixadora de nitrogênio Bradyrhizobium japonicum, optou-se neste estudo pela investigação do efeito do conteúdo de fósforo, nutriente de comum deficiência nos solos brasileiros, sobre o sistema radicular da planta, com a finalidade de desenvolver a metodologia proposta.
O objetivo deste trabalho foi estabelecer uma metodologia para utilizar a modelagem matemática como ferramenta para agregar dados experimentais coletados, que represente os processos definidores da arquitetura radicular, e apresente-os de uma maneira em que comparações entre tratamentos (níveis de P disponível no solo, neste caso) possam ser feitas. O modelo apresentado não tem o intuito de ser utilizado em cenários de previsão.

\section{MATERIAL E MÉTODOS}

\section{Terminologia}

\section{Descrição de classes de desenvolvimento}

Não existe atualmente uma padronização na classificação dos segmentos de um sistema radicular (ramos) quanto à sua ordem de desenvolvimento. Visando uma uniformização de linguagem, sugere-se que a classificação proposta por Rose (1983) passe a ser adotada na prática, visto ser a mais conveniente e de fácil entendimento.

Rose (1983) estabelece que: (i) as raízes produzidas diretamente a partir da base da planta são denominadas eixos (uma monocotiledônea pode ter vários eixos em seu sistema radicular, enquanto que uma dicotiledônea com sistema radicular pivotante possui apenas um eixo), (ii) as raízes laterais que emergem do(s) eixo(s) são denominadas raízes laterais de primeira ordem, (iii) as raízes laterais que emergem das laterais de primeira ordem são denominadas laterais de segunda ordem, e assim por diante. O mesmo autor ainda estabelece a denominação ordem 0 para os eixos, ordem 1 para as raízes laterais de primeira ordem, ordem 2 para as raízes laterais de segunda ordem, etc.

\section{Procedimento experimental}

Plantas de soja (Glycine max L. Merrill cv. Williams 82) foram cultivadas em caixas de acrílico preto, com paredes laterais inclinadas e deslizáveis de acrílico transparente, que permitiam a observação do crescimento e desenvolvimento das raízes. Tais paredes transparentes foram cobertas por placas deslizáveis de acrílico preto, para evitar a incidência de luz sobre os sistemas radiculares. Cada caixa de acrílico possuía dois compartimentos com as seguintes dimensões: $35 \mathrm{~cm}$ de altura, $39 \mathrm{~cm}$ de comprimento, $7 \mathrm{~cm}$ de largura no topo e $2 \mathrm{~cm}$ de largura no fundo (Figura 1). Em cada compartimento foi depositada apenas uma semente. A superfície exposta do substrato foi coberta com papel alumínio logo após a emergência das plantas, para evitar evaporação. Logo após a semeadura, as caixas foram colocadas em câmara de crescimento. A temperatura do ar dentro da câmara de crescimento foi mantida variando entre um valor máximo diário de $27^{\circ} \mathrm{C}$ e um mínimo diário 
de $19^{\circ} \mathrm{C}$, com fotoperíodo constante de 15 horas durante todo o período experimental, de até 30 dias. As caixas foram preenchidas com substrato inerte seco, com concentração insignificante de fósforo, constituído de uma mistura 1:1 em volume de areia (mesh 41) e "Sunshine mix \#1" (Sun Gro Horticulture Inc.), composto de 70 a $80 \%$ de esfagno, além de perlita, gesso, agente molhante e calcário dolomítico para ajuste do $\mathrm{pH}$. O conteúdo desejado de nutrientes foi conseguido pela adição de solução nutritiva até um conteúdo volumétrico de água de $0,34 \mathrm{~cm}^{3} \mathrm{~cm}^{-3}$, atingindo concentração uniforme e ideal de todos os nutrientes, variando apenas a concentração de fósforo, de acordo com os dois tratamentos utilizados (alta concentração de fósforo e baixa concentração de fósforo, aqui designadas por $+\mathrm{P}$ e $-\mathrm{P}$, respectivamente). A massa específica do substrato dentro das caixas foi de $1,23 \mathrm{~g} \mathrm{~cm}^{-3}$ para ambos os tratamentos. A composição da solução nutritiva foi a seguinte: $1,67 \mathrm{mmol} \mathrm{L}^{-1} \mathrm{KH}_{2} \mathrm{PO}_{4}, 5$ $\mathrm{mmol} \mathrm{L}{ }^{-1} \mathrm{KNO}_{3}, 5 \mathrm{mmol} \mathrm{L}^{-1} \mathrm{Ca}\left(\mathrm{NO}_{3}\right)_{2}$, e $2 \mathrm{mmol} \mathrm{L}^{-1} \mathrm{MgSO}_{4}$ para o tratamento $+\mathrm{P}$, e $0,33 \mathrm{mmol} \mathrm{L}^{-1} \mathrm{KH}_{2} \mathrm{PO}_{4}, 6,67 \mathrm{mmol}$ $\mathrm{L}^{-1} \mathrm{KNO}_{3}, 5 \mathrm{mmol} \mathrm{L}^{-1} \mathrm{Ca}\left(\mathrm{NO}_{3}\right)_{2}$, e $2 \mathrm{mmol} \mathrm{L}^{-1} \mathrm{MgSO}_{4}$ para o tratamento -P; em ambas as soluções, os micronutrientes foram adicionados nas seguintes concentrações: $0,5 \mathrm{mg} \mathrm{L}^{-1} \mathrm{~B}, 0,5 \mathrm{mg} \mathrm{L}^{-1} \mathrm{Mn}, 0,05 \mathrm{mg} \mathrm{L}^{-1}$ $\mathrm{Zn}, 0,02 \mathrm{mg} \mathrm{L}^{-1} \mathrm{Cu}$, e $0,01 \mathrm{mg} \mathrm{L}^{-1} \mathrm{Mo}$ (solução de Hoagland e Arnon, de acordo com Gauch, 1972). Embora a concentração de $P$ nas soluções fosse de $51,6 \mathrm{mg} \mathrm{L}^{-1}$, e $10,3 \mathrm{mg} \mathrm{L}^{-1}$ para os tratamentos $+\mathrm{P}$ e $-\mathrm{P}$, respectivamente, a concentração inicial alcançada nas caixas, após adsorção pelo substrato sólido, foi de $15,5 \mathrm{mg} \mathrm{L}^{-1} \mathrm{P}$, e $8,5 \mathrm{mg} \mathrm{L}^{-1} \mathrm{P}$ (pelo método Bray-P).

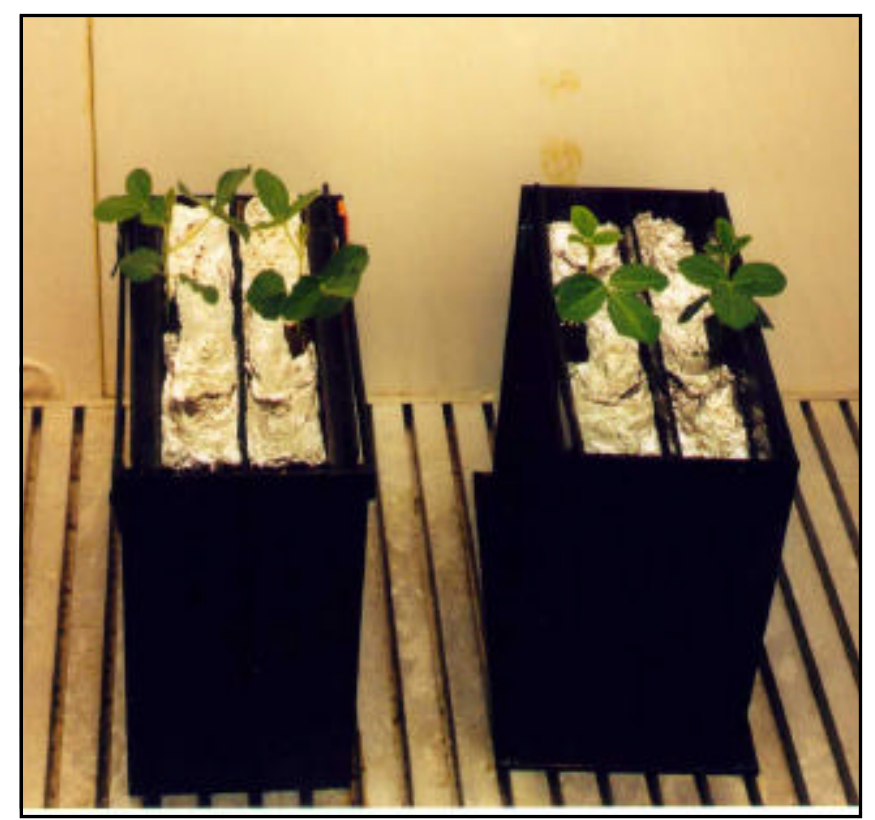

\section{Descrição do procedimento de medida do sistema radicular}

Antes de cada amostragem, os sistemas radiculares eram observados através das paredes de acrílico transparente e realizavam-se medidas diretas dos ângulos de orientação e ramificação.

Para a obtenção dos valores de densidade de ramificação e comprimento dos trechos radiculares da raiz principal e das raízes laterais de ordens 1 a 3, adotou-se um processo de análise destrutivo, no qual as plantas foram removidas do solo a cada 5 dias $(5,10,15,20,25$ e 30 dias após emergência), sendo seu sistema radicular meticulosamente medido com uma régua flexível.

Em cada amostragem, o sistema radicular foi retirado do substrato de maneira a se evitar o rompimento de algum trecho do mesmo, sendo em seguida lavado por imersão em água antes de iniciar-se o procedimento de medida.

No procedimento de medida utilizado em todas as amostragens, a raiz principal pivotante (ordem 0 ) tinha seu comprimento determinado, após o que o sistema radicular era dividido em trechos de $5 \mathrm{~cm}$ de comprimento ao longo da raiz principal. Eram então contados os números de raízes laterais de ordem $1 \mathrm{em}$ cada trecho de $5 \mathrm{~cm}$ da raiz principal e em seguida 6 raízes de ordem 1 eram retiradas de cada trecho, completamente ao acaso, e tinham seu comprimento determinado, com a finalidade de estimativa do comprimento médio de acordo com sua posição de origem na raiz principal. Em seguida eram escolhidos 6 trechos de $3 \mathrm{~cm}$ ao longo das raízes de ordem 1

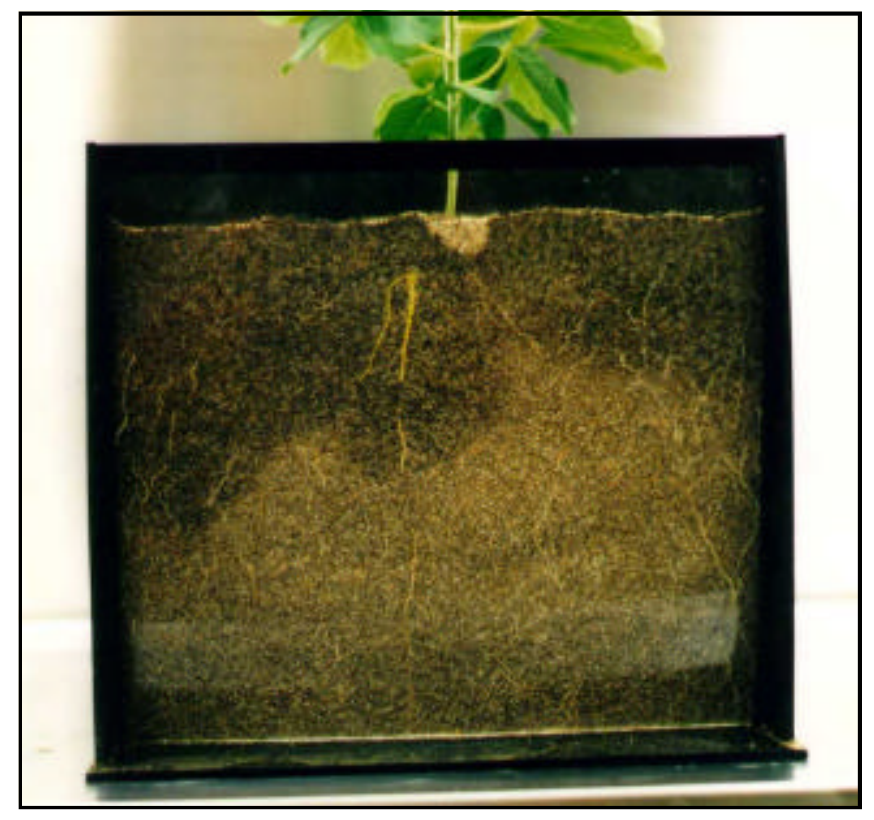

Figura 1 - Caixas de acrílico preto com paredes laterais inclinadas de acrílico transparente cobertas por placas deslizáveis de acrílico preto. $\mathrm{Na}$ foto à esquerda observa-se a superfície exposta do substrato coberta por papel alumínio para diminuir a evaporação. Na foto à direita observa-se, através da parede transparente da caixa, as raízes crescendo no substrato. 
identificadas de acordo com sua posição de origem, e era contado o número e medido o comprimento das raízes de ordem 2, para estimativa do número médio de raízes de ordem 2 por centímetro de raiz de ordem 1 , e seu comprimento médio. O mesmo procedimento era adotado para as raízes de ordem 3 , quando existentes.

Sendo assim, para cada tratamento, idade de amostragem e repetição foram obtidos: (i) comprimento da raiz principal (ordem 0), e (ii) de acordo com a posição de origem na raiz principal, os respectivos números e comprimentos médios das raízes laterais das ordens 1,2 e 3 .

Como o delineamento experimental contava com 3 repetições para cada idade de planta avaliada $(5,10$, $15,20,25$, e 30 dias após a germinação), também foram calculados os valores médios das 3 repetições.

\section{Desenvolvimento do modelo}

A arquitetura radicular é um agregado de características geradas por diversos processos distintos que ocorrem em cada segmento radicular, sendo que apenas alguns são conhecidos e entendidos, sendo os principais, segundo Lynch (1995): (i) o crescimento em extensão de segmentos radiculares individuais, (ii) 0 aparecimento de raízes filhas sobre cada segmento radicular, (iii) a direção do crescimento de cada segmento radicular, (iv) a senescência ou mortalidade dos segmentos radiculares, e (v) a plasticidade (adaptabilidade) desses processos em resposta a diferentes condições edafoclimáticas, tais como resistência do solo à penetração, disponibilidade de nutrientes e conteúdo de água e oxigênio do solo.

Desenvolveram-se algoritmos representando cada processo definidor do crescimento e arquitetura radiculares, na linguagem de programação Visual Basic para Windows, de maneira a gerar uma imagem simulada que representasse o sistema radicular real que alimentou o modelo. A listagem das rotinas computacionais do modelo desenvolvido está à disposição dos leitores mediante solicitação aos autores.

\section{RESULTADOS E DISCUSSÃO}

\section{Atributos do sistema radicular e parâmetros do modelo}

\section{Comprimento dos ramos}

Denomina-se ramo um segmento radicular localizado entre o ponto de origem (semente, parte aérea ou ponto de ramificação) e seu respectivo meristema apical. Curvas de comprimento médio do ramo (CR) foram ajustadas através de análise de regressão para cada ordem e profundidade de origem (de $5 \mathrm{em} 5 \mathrm{~cm}$ ), e para os dois tratamentos de $\mathrm{P}$ no solo ( $15 \mathrm{ppm}$ e $8 \mathrm{ppm}$ ), em função da idade da planta, utilizando-se a seguinte equação:

$$
C R=\frac{a}{1+\left(\frac{D A G}{b}\right)^{c}}
$$

a, b e c = parâmetros de ajuste; DAG = idade da planta, em dias após a germinação

O ajuste foi feito em função da idade da planta e não da idade do ramo por uma questão de adequação ao modelo de simulação da arquitetura radicular que foi desenvolvido.

As curvas de comprimento médio dos ramos de todas as ordens, para algumas profundidades escolhidas, são apresentadas na Figura 2. A maioria das curvas ajustadas teve valores de coeficiente de regressão acima de 0,95. Não se apresentam os parâmetros de ajuste ( $a, b$ e $c$ ) para cada curva, pois tais curvas ajustadas não são extrapoláveis, sendo válidas apenas para o sistema radicular estudado. Embora tenha-se utilizado e sugerido uma equação específica para a análise de regressão, qualquer outra equação que produza curvas sigmóides pode ser utilizada no ajuste de curvas, desde que a mesma equação seja utilizada para todas as ordens e profundidades.

\section{Velocidade de Crescimento}

O termo "velocidade de crescimento" descreve o parâmetro de acréscimo médio diário no comprimento de um ramo (em centímetros por dia).

A velocidade de crescimento (VC) é um parâmetro dependente de vários fatores edafoclimáticos, como temperatura e umidade do solo, resistência do solo à penetração e características químicas do solo, entre outros, além de fatores da planta, como genótipo, idade do ramo, ordem de desenvolvimento do ramo (raiz principal pivotante, de ordem 0 , ou raízes laterais, de ordem 1, 2, ou 3, no caso da soja), e, para as raízes laterais, profundidade de origem do ramo de ordem 1 ou profundidade do ponto de conexão com a raiz principal para as ramificações de ordens 2 e 3 .

As curvas de VC foram obtidas a partir das derivadas das equações ajustadas de comprimento médio de ramo (equação 2), sendo apresentadas na Figura 3.

$$
V C=\frac{-a \cdot\left[c \cdot\left\{\left(\frac{1}{b}\right)^{c} \cdot D A G^{c-1}\right\}\right]}{\left\{1+\left(\frac{D A G}{b}\right)^{c}\right\}^{2}}
$$

\section{Distância de Ramificação}

O número de ramos de todas as ordens foi medido, sendo calculados os valores médios e os seus desvios padrão. Tais valores foram utilizados no cálculo de Distância de Ramificação.

Verificou-se variação significativa na distância entre dois pontos de ramificação, aqui denominada Distância de Ramificação (DR), em função da profundidade 

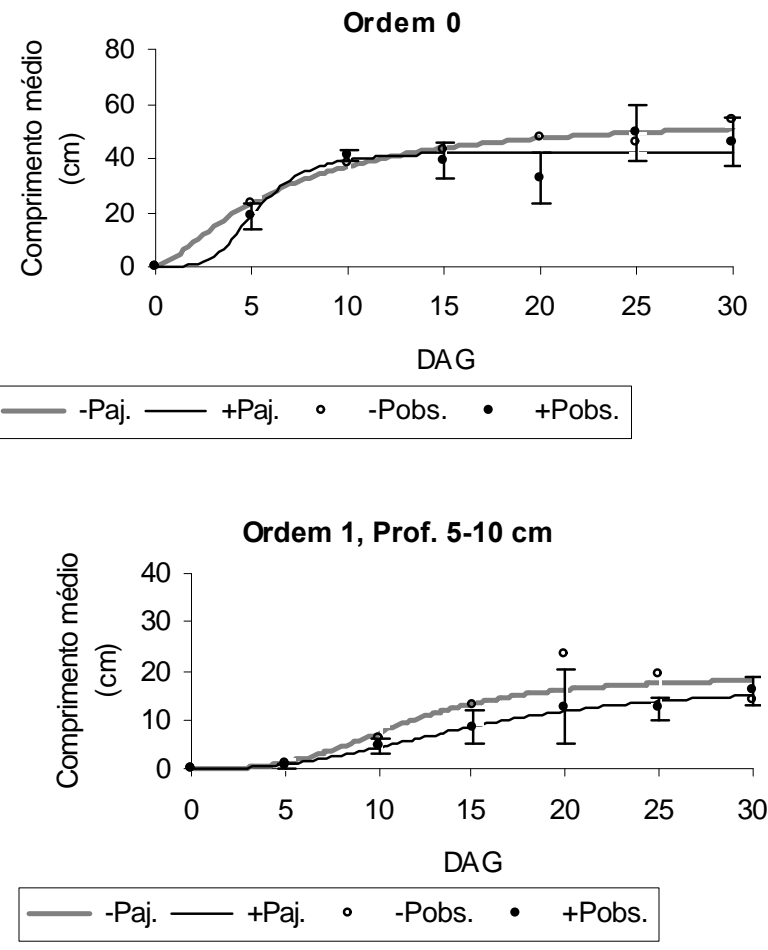

Ordem 2, Prof. 0-5 cm

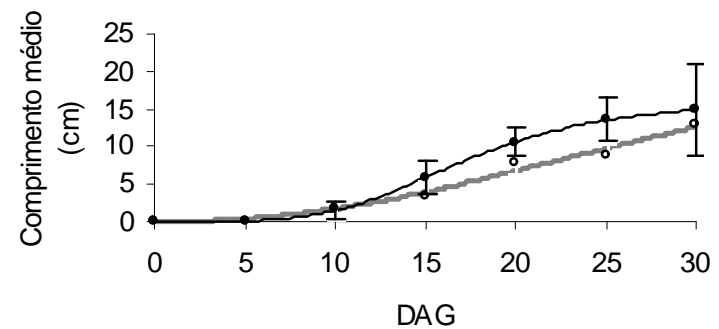

-Paj. -+ +Paj. $\quad$ ○ -Pobs. $\bullet$ +Pobs.

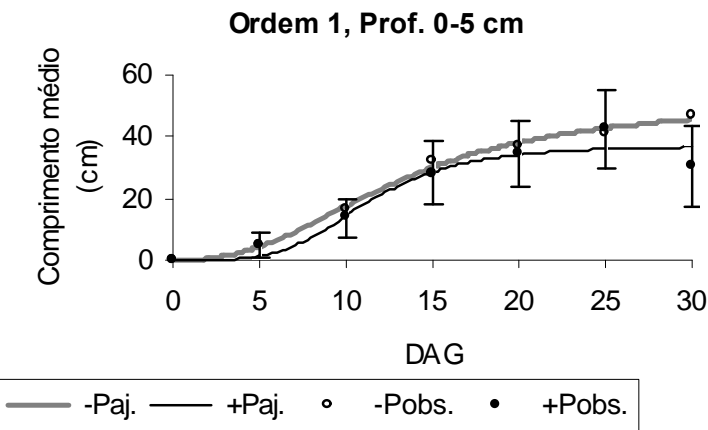

Ordem 1, Prof. 25-30 cm

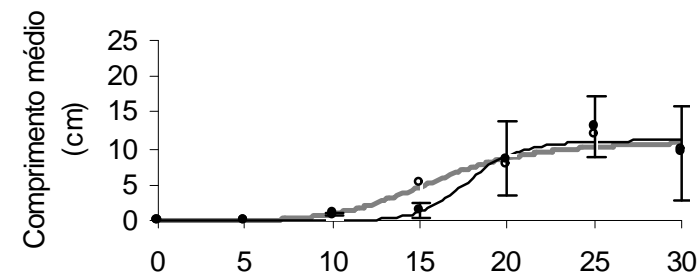

DAG

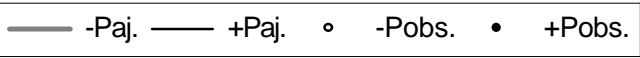

Ordem 3, Prof. 0-5 cm

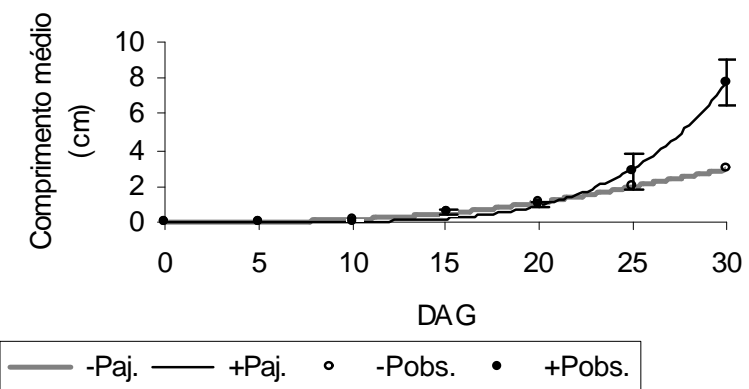

Figura 2 - Curvas ajustadas de comprimento médio do ramo (-Paj. e +Paj., baixo e alto teor de P, respectivamente) para raízes de ordens 0 a 3, para algumas profundidades de origem selecionadas. Pontos (-Pobs. e +Pobs., 8,5 e 15,5 mg L-1 de $P$, respectivamente) representam valores medidos experimentalmente (médias de três repetições), junto com as barras de desvio padrão. DAG = dias após a germinação.

de origem (ramificações sobre a raiz de ordem 0), ou em função da profundidade de conexão com a raiz de ordem 0 (ramificações sobre as raízes de ordens 1 e 2). Não se verificou ramificação sobre as raízes de ordem 3 . As curvas de DR para os dois tratamentos de $\mathrm{P}$ no solo (Figura 4) foram obtidas através de análise de regressão, sendo ajustadas à seguinte equação:

$$
D R=d+e \cdot V P^{2}+f \cdot V P^{2} \cdot \log (V P)+g \cdot \operatorname{Exp}(V P)
$$

$\mathrm{d}$, e, f, $\mathrm{g}=$ parâmetros de ajuste; $V P=$ variável de profundidade, sendo igual à profundidade de origem, para as ramificações sobre a raiz de ordem 0 , ou igual à profundidade de conexão com a raiz de ordem 0 , para as ramificações sobre as raízes de ordens 1 e 2 .

Como no caso das curvas de comprimento dos ramos, não se apresentam os parâmetros de ajuste (d, e, f, g), pelas mesma razões já descritas. Qualquer outra equação que explique melhor os pontos experimentais coletados pode ser usada na análise de regressão.

\section{Duração da não ramificação do ápice}

Essa terminologia utilizada em modelos bem conceituados, como o de Diggle (1988), transmite a idéia errônea de que o ápice dos ramos radiculares poderia se ramificar. Um potencial ponto de ramificação (dado pela DR) começa a desenvolver um novo ramo a partir de uma idade determinada. O fenômeno é representado adequadamente no presente modelo pelo ajuste das curvas de VC em função da idade da planta e para cada profundidade de origem (verificar na Figura 3 como algumas curvas de VC não partem do zero na idade 0 , mas em épocas posteriores). 

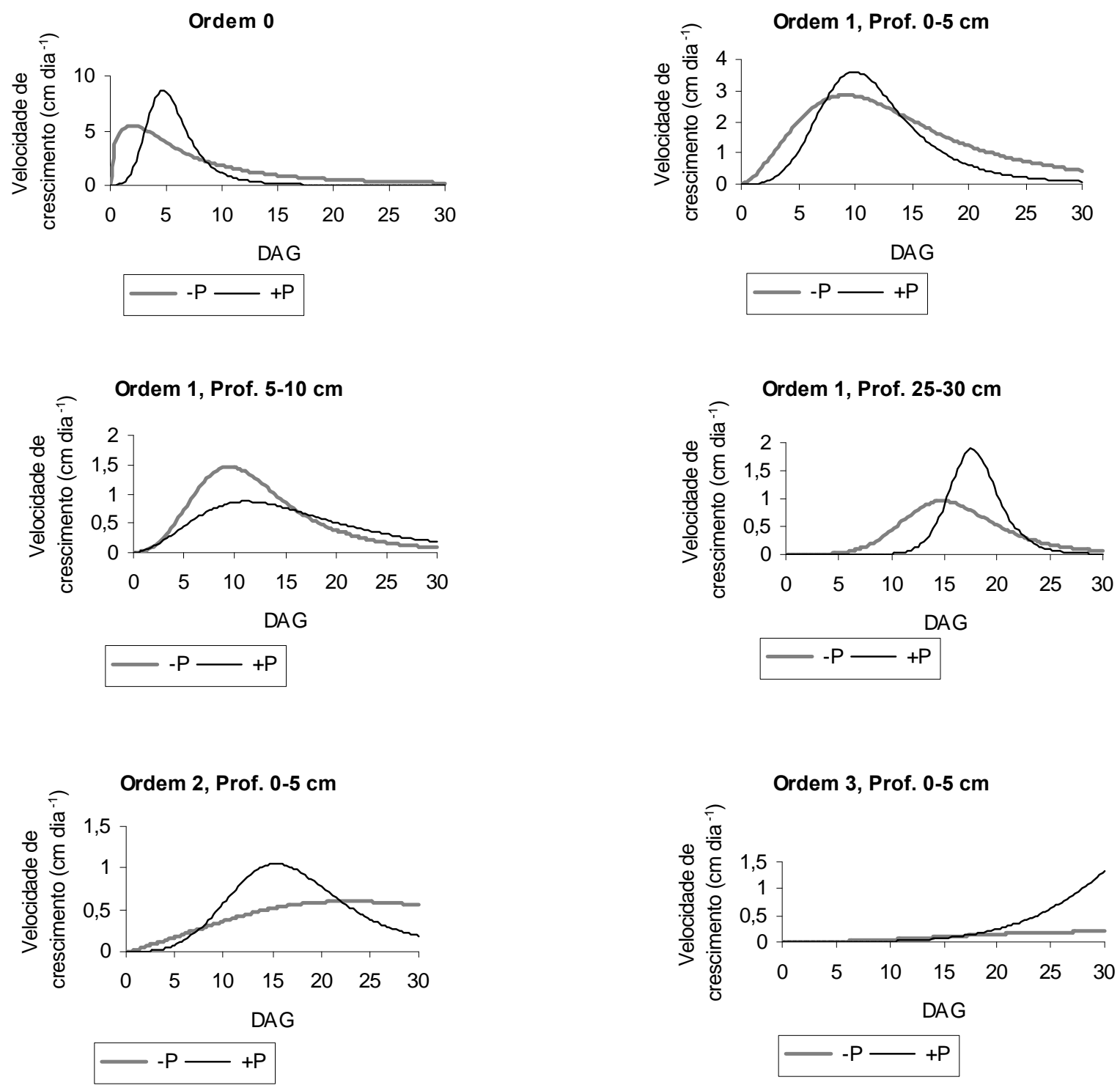

Figura 3 - Variação temporal da velocidade de crescimento do ramo para raízes de ordens 0 a 3 , para algumas profundidades de origem selecionadas. $-\mathrm{P}$ representa $\mathrm{o}$ tratamento com baixo teor de $\mathrm{P}$ no substrato $\mathrm{e}+\mathrm{P}$ o tratamento com alto teor de $\mathrm{P}$ no substrato. Curvas são derivadas das curvas de comprimento médio do ramo apresentadas na Figura 2. DAG = dias após a germinação.

Parâmetros afetando a direção de crescimento

A cada novo incremento no comprimento de um ramo, a direção na qual a ponta da raiz cresce segue um ângulo de orientação, e pode defletir aleatoriamente dentro de uma faixa estabelecida. O ângulo de orientação depende da reação gravitrópica do ramo, ou seja, sua preferência de crescimento num ângulo vertical. A reação gravitrópica, por sua vez, depende da ordem de desenvolvimento do ramo e de sua idade. Na cultura de soja, o ramo de ordem 0 (raiz principal pivotante) tem uma alta reação gravitrópica, ou seja, cresce num ângulo estritamente vertical, apresentando apenas pequenas deflexões durante seu crescimento. Os ramos de ordem 1 (raízes laterais de ordem 1) não apresentam nenhuma reação gravitrópica quando surgem, aumentando essa reação com o aumento de sua idade. Os ramos de ordens 2 e 3 não apresentam reação gravitrópica em nenhuma fase de sua vida, ou seja, surgem em ângulos perpendiculares à raiz mãe e apresentam apenas variações deflectivas.

O presente modelo não usa como parâmetros Índices de Geotropismo (Gravitropismo) e Deflexão, como a maioria dos modelos existentes, mas sim ângulos de orientação, com possibilidade de variação aleatória dentro de uma faixa (faixa de deflexão). Sendo assim, obteve-se os seguintes valores experimentais: a raiz principal pivotante cresce verticalmente $\left(0^{\circ} \mathrm{com}\right.$ a normal), podendo defletir em até mais ou menos $10^{\circ}$ durante cada novo acréscimo no comprimento. As raízes laterais de ordem 1 se originam perpendicularmente à raiz de ordem $0\left(90^{\circ}\right.$ 

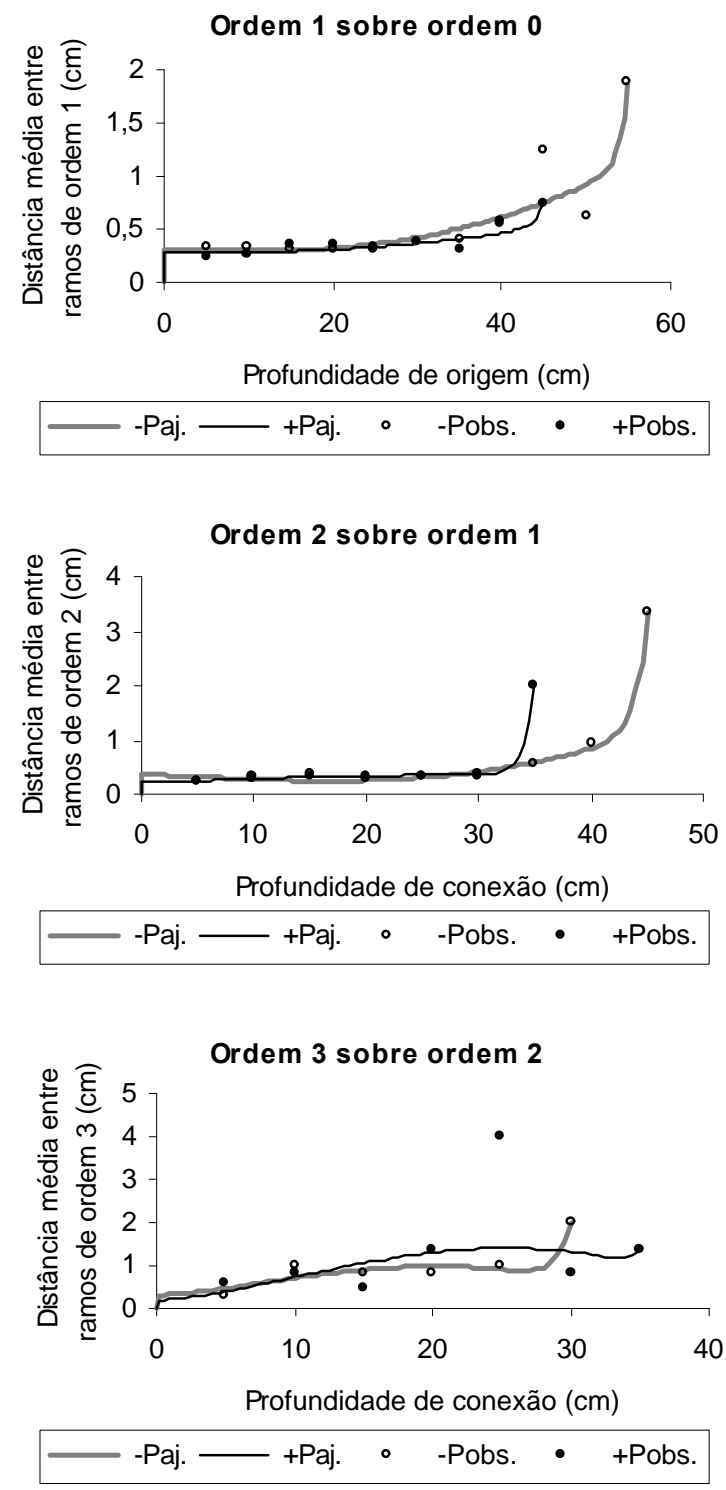

Figura 4 - Curvas ajustadas de Distância de Ramificação (-Paj. e + Paj., baixo e alto teor de $P$, respectivamente). Pontos (-Pobs. e +Pobs., 8,5 e 15,5 $\mathrm{mg} \mathrm{L}^{-1}$ de $\mathrm{P}$, respectivamente) representam valores medidos experimentalmente (médias de três repetições).

com a normal) e apresentam ângulos progressivamente menores em relação à normal a cada acréscimo de comprimento $\left(90^{\circ}\right.$ até $1 \mathrm{~cm}, 65^{\circ}$ de 1 a $5 \mathrm{~cm}, 55^{\circ}$ de 5 a $10 \mathrm{~cm}, 35^{\circ}$ de 10 a $15 \mathrm{~cm}, 25^{\circ}$ de 15 a $20 \mathrm{~cm}, 15^{\circ}$ de 20 a $25 \mathrm{~cm}, 5^{\circ}$ de 25 a $30 \mathrm{~cm}$, e $0^{\circ}$ a partir de $30 \mathrm{~cm}$ de distância da raiz principal), podendo ainda apresentar uma deflexão de até mais ou menos $15^{\circ}$ a cada novo trecho crescido. As raízes laterais de ordens 2 e 3 se originam perpendicularmente em relação à sua raiz mãe e podem apresentar deflexão de até mais ou menos $40^{\circ}$ a cada novo trecho crescido.

Não se verificou influência significativa da concentração de fósforo no substrato sobre os ângulos de orientação das raízes laterais de ordem 1 .

\section{A simulação}

A análise de regressão, e a comparação de médias e desvios padrão, integrantes da estatística clássica, são úteis para explicar diferenças em segmentos individuais do sistema radicular, como visto nas Figuras 2 a 4. No entanto, tais técnicas não permitem que se compare diferentes arquiteturas radiculares e se explique as diferentes estratégias adaptativas adotadas pelas plantas em face de restrições edafoclimáticas.

A modelagem matemática, por outro lado, permite a agregação de todos os atributos e processos radiculares e o cálculo de sua influência sobre o sistema radicular inteiro, permitindo a visualização de um sistema radicular simplificado, mas representativo, e a comparação entre diferentes arquiteturas radiculares.

É praticamente impossível detectar variações arquiteturais em sistemas radiculares sujeitos a pequenas variações edáficas olhando para os sistemas radiculares reais. Portanto, o modelo proposto integra os parâmetros e processos radiculares e produz uma representação dinâmica bidimensional do sistema radicular estudado. Tais imagens simuladas não são previsões de arquitetura radicular em resposta a um conjunto de fatores edafoclimáticos, mas simplesmente a reprodução, de forma esquemática e simplificada, dos sistemas radiculares reais observados.

Embora imagens tridimensionais possam aumentar o realismo e serem mais adequadas para integração com modelos de absorção de água e solutos, imagens em duas dimensões, como simuladas pelo modelo proposto, são mais apropriadas para a caracterização de sistemas radiculares através de comparações visuais, permitindo assim a identificação de estratégias adaptativas.

Os algoritmos representando os processos de formação e crescimento de raízes laterais de ordem 3 estavam inativos no modelo durante a simulação, visto que a capacidade dos microcomputadores disponíveis para este estudo (Processador Pentium II, 400 MHz; Memória $\mathrm{RAM}=64 \mathrm{Mb}$ ) foi insuficiente.

Apresentam-se as imagens simuladas para os dois tratamentos, a intervalos de 5 dias, até 30 dias após a germinação, na Figura 5.

Cabe esclarecer o significado de dois termos: "exploração do solo" e "utilização do solo". O primeiro representa o volume de solo explorado pelo sistema radicular de uma planta. O segundo representa a quantidade de nutrientes ou água absorvidos por massa ou volume de raiz produzidos.

Uma maior eficiência de exploração é conseguida por um crescimento acelerado da raiz principal, de ordem 0 , e das raízes basais (raízes laterais de ordem 1 que se desenvolvem nos $5 \mathrm{~cm}$ superficiais do solo e que apresentam um desenvolvimento bastante pronunciado em relação às demais). Para nutrientes imóveis no solo, como o fósforo, uma maior eficiência de utilização é conseguida 

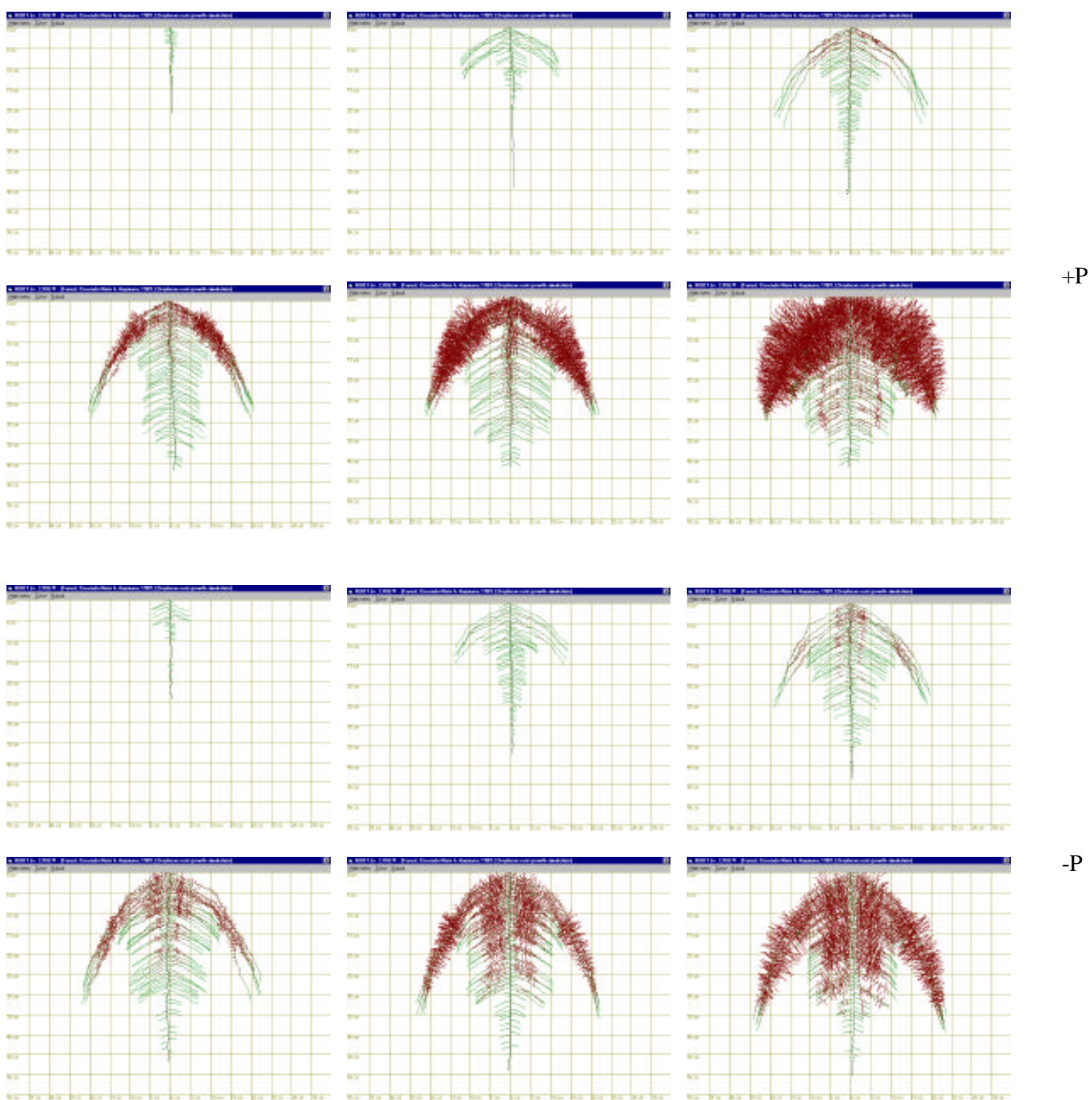

Figura 5 - Representação esquemática da dinâmica do sistema radicular de soja para concentrações de fósforo no solo alta (+P) e baixa (-P), para 5, 10, 15, 20, 25 e 30 dias após a germinação. Quadriculados representam uma distância de 5 por $5 \mathrm{~cm}$.

por um sistema radicular que apresente uma maior freqüência de ramificação de raízes finas (menor distância de ramificação) em zonas de maior fertilidade no solo.

Verificou-se na condição de menor concentração de fósforo no solo, uma maximização da exploração do solo, na procura pelo recurso escasso, enquanto que na condição de maior concentração de fósforo no solo, houve uma maximização da utilização do solo, para uso do recurso existente.

Conforme esperado, os dados experimentais e a representação dinâmica do sistema radicular produzida pelo modelo mostram uma alocação maior de fotossintatos para as raízes de ordem 0 e basais de ordem 1 (responsáveis pela exploração do solo em busca do recurso escasso) no tratamento com menor teor de $\mathrm{P}$ no solo, e uma alocação maior de fotossintatos para as raízes de ordem 2, de maior capacidade de absorção (responsáveis pela utilização do recurso disponível no solo) no tratamento com maior teor de $\mathrm{P}$ no solo.

A aparência arquitetural do sistema radicular em resposta à concentração de fósforo no solo representa uma estratégia adaptativa da planta para a obtenção da quantidade necessária deste recurso gastando o mínimo possível de fotoassimilados para crescimento e formação de estruturas radiculares (Nielsen et al., 1994; Lynch, 1995). 


\section{CONSIDERAÇÕES FINAIS}

O modelo matemático de simulação é uma ferramenta útil no teste de hipóteses derivadas de resultados experimentais, permitindo um aumento na capacidade de entendimento de fenômenos naturais. A modelagem matemática representa uma promissora metodologia de análise de dados de sistemas radiculares, possibilitando uma adequada visualização, quantificação e interpretação de diferentes estratégias adaptativas da planta, conforme refletidas pelas diferentes arquiteturas radiculares produzidas em cada condição edafoclimática. À medida em que o modelo permite a identificação de pontos cujo conhecimento e entendimento ainda é falho, ele pode ser utilizado na definição de prioridades de pesquisa e para ressaltar a necessidade de trabalho multidisciplinar em certas áreas. Todos os modelos matemáticos, inclusive o objeto deste trabalho, são mais úteis pelo discernimento que eles nos proporcionam do que por sua habilidade de prever fenômenos naturais (Wullschleger et al., 1994).

O suprimento de água e nutrientes das plantas depende das interações entre os complexos processos fisiológicos e celulares ocorrentes no sistema radicular, que resultam numa arquitetura específica (habilidade da planta adquirir os recursos do solo), e dos não menos complexos processos iônicos e de transporte ocorrentes no solo (disponibilidade dos recursos do solo às raízes). $\mathrm{O}$ atual conhecimento e entendimento de tais processos e de suas interações ainda é insatisfatório, tornando-se urgente a união de forças entre pesquisadores trabalhando com fisiologia do crescimento radicular, caracterização experimental de sistemas radiculares, física, química e biologia de solos e profissionais trabalhando com modelagem matemática.

Sugere-se o emprego da metodologia aqui proposta em estudos futuros dos efeitos de outras variáveis, como a temperatura, a umidade e a resistência à penetração do solo sobre a aparência do sistema radicular da soja ou de outra espécie dicotiledônea herbácea ou arbórea.

\section{AGRADECIMENTOS}

À Fundação de Amparo à Pesquisa do Estado de São Paulo (FAPESP) e ao United States Department of Agriculture (USDA) pelo apoio financeiro concedido, ao Dr. T. C. Hsiao, da University of California, Davis, EUA pela colaboração no delineamento experimental, e à Dra. F. Somma, do EC - Joint Research Centre/ Space Applications Institute, Itália, pelas discussões e sugestões apresentadas.

\section{REFERÊNCIAS BIBLIOGRÁFICAS}

CLAUSNITZER, V.; HOPMANS, J. W. An algorithm for three dimensional, simultaneous modeling of root growth and transient soil water flow (version 1.0). Davis: LAWR/ UCDAVIS, 1993. 108p.

DIGGLE, A.J. ROOTMAP: a model in three-dimensional coordinates of the growth and structure of fibrous root systems. Plant and Soil, v.105, p.169-178, 1988.

DREW, M.C. Comparison of the effects of a localized supply of phosphate, nitrate, ammonium and potassium on the growth of the seminal root system, and the shoot, in barley. New Phytologist, v.75, p.479-490, 1975.

GAUCH, H. G. Inorganic plant nutrition. Stroudsburg: Dowden, Hutchinson \& Ross, 1972. 488p.

HACKETT, C.; ROSE, D. A. A model of the extension and branching of a seminal root of barley, and its use in studying relations between root dimensions: 1. The model. Australian Journal of Biological Sciences, v.25, p.669-679, 1972.

LUNGLEY, D. R. The growth of root systems: a numerical computer simulation model. Plant and Soil, v.38, p.145-159, 1973.

LYNCH, J. Root architecture and plant productivity. Plant Physiology, v.109, p.7-13, 1995.

LYNCH, J.P.; NILSEN, K.L.; DAVIS, R.D.; JABLOKOW, A. G. SimRoot: modelling and visualization of root systems. Plant and Soil, v.188, p.139-151, 1997

McCULLY, M. How do real roots work? Some new views of root structure. Plant Physiology v.109, p.1-6, 1995.

NIELSEN, K.L.; LYNCH, J.; JABLOKOW, A.G.; CURTIS, P.S. Carbon cost of root systems: an architectural approach. Plant and Soil, v.165, p.161-169, 1994.

PAGES, L.; JORDAN, M. O.; PICARD, D. A simulation model of the three-dimensional architecture of the maize root system. Plant and Soil, v.119, p.147-154, 1989.

PORTER, J. R.; KLEPPER, B.; BELFORD, R. K.; A model (WHTROOT) which synchronizes root growth and development with shoot development for winter wheat. Plant and Soil, v.92, p.133-145, 1986.

ROBINSON, D. Roots and resource fluxes in plants and communities. In: ATKINSON, D. (Ed.) Plant root growth: an ecological perspective. Oxford: Blackwell, 1991. p.103-130.

ROBINSON, D. The responses of plants to non-uniform supplies of nutrients. New Phytologist, v.127, p.635-674, 1994.

ROSE, D. A. The description of the growth of root systems. Plant and Soil, v.75, p.405-415, 1983.

SOMMA, F.; HOPMANS, J.W.; CLAUSNITZER, V. Transient three-dimensional modeling of soil water and solute transport with simultaneous root growth, root water and nutrient uptake. Plant and Soil, v.202, p.281-293, 1998.

WULLSCHLEGER, S.D.; LYNCH, J.P.; BERNTSON, G.M. Modeling the belowground response of plants and soil biota to edaphic and climatic change - What can we expect to gain? Plant and Soil, v.165, p.149-160, 1994.

Recebido em 20.09.99 